\title{
A compact hexapole state-selector for NO radicals
}

\author{
Moritz Kirste, ${ }^{1}$ Henrik Haak, ${ }^{1}$ Gerard Meijer, ${ }^{1}$ and Sebastiaan Y.T. van de Meerakker ${ }^{2,1}$ \\ 1) Fritz-Haber-Institut der Max-Planck-Gesellschaft, Faradayweg 4-6, 14195 Berlin, \\ Germany \\ ${ }^{2)}$ Radboud University Nijmegen, Institute for Molecules and Materials, Heijendaalseweg 135, 6525 AJ Nijmegen, \\ The Netherlands
}

(Dated: 23 April 2013)

Focusing of molecular beams using an electrostatic hexapole is a mature technique to produce samples of state-selected molecules. The ability to efficiently focus molecules depends on the properties of the molecular species of interest, the length of the hexapole state selector, as well as on the maximum electric field strength that can be achieved in these devices. In particular for species with a small effective dipole moment such as nitric oxide (NO), hexapole state selectors of several meters in length are required to focus the beam. We report on a novel design for an electrostatic hexapole state-selector that allows for a maximum electric field strength of $260 \mathrm{kV} / \mathrm{cm}$, reducing significantly the length of the hexapole that is required to focus the beam. We demonstrate the focusing of a molecular beam of NO radicals $\left(X^{2} \Pi_{1 / 2}, v=0, J=1 / 2, f\right)$ using a hexapole of only $30 \mathrm{~cm}$ length. A beamstop is integrated inside the hexapole at the geometric center of the device where the molecular trajectories have the largest deviation from the beam axis, effectively blocking the carrier gas of the molecular beam at minimum loss of NO density. The performance of the hexapole stateselector is investigated by state-selective laser induced fluorescence detection, as well as by two dimensional imaging of the focused packet of NO radicals. The resulting packet of NO radicals has a density of $9 \pm 3 \cdot 10^{10}$ $\mathrm{cm}^{-3}$ and a state purity of $99 \%$.

\section{INTRODUCTION}

The technique of state-selection and focusing of molecular beams using electrostatic hexapoles has been widely used in molecular beam research over the last five decades, and has found many applications ${ }^{1}$. It was pioneered by the groups of Bernstein and Brooks already in the $1960 \mathrm{~s}^{2-4}$, who realized that a hexapolar field geometry is advantageous for molecular species with a linear Stark shift over the - at the time better known quadrupolar geometry. Originally, their work aimed at observing and understanding steric effects in chemical reactions at the molecular level ${ }^{5}$. Over the years, electrostatic as well as magnetic hexapoles ${ }^{6,7}$ have been applied in various experiments, producing molecular beams with almost perfect state selection ${ }^{8-12}$.

An important application of hexapole state selection is found in the investigation of inelastic or reactive collisions of polar molecules with atoms or other molecules. In these experiments, the preparation of molecules in a single quantum state prior to the collision using a hexapole allows for the measurement of state-to-state cross sections and steric effects. Crossed molecular beam machines employing hexapole state selectors have been implemented in many laboratories, and the wealth of scattering studies that have been carried out has lead to the understanding of propensity rules for rotational energy transfer ${ }^{13}$, the stereodynamics of molecular collisions ${ }^{14}$ and reactivity ${ }^{15}$, as well as quantum interference effects ${ }^{16}$. In particular the open-shell radical species $\mathrm{NO}^{17-19}$ and $\mathrm{OH}^{20-23}$ have been frequently used in such experiments, benchmarking our present understanding of how intermolecular potentials govern molecular collision dynamics ${ }^{24-28}$. Overview articles about the state-selection of $\mathrm{OH}$ and $\mathrm{NO}$ radicals using electrostatic hexapoles are found in Hain et al. ${ }^{29}$ and Bichsel et al. ${ }^{30}$, respectively.

Hexapoles have also been used extensively to study the interactions between molecules and surfaces, which has strongly influenced the field of surface science ${ }^{9}$. The molecule $\mathrm{CF}_{3} \mathrm{H}$ was state-selected and scattered from a $\mathrm{Ag}$ surface ${ }^{31}$, revealing a dependency of the orientation of the molecules leaving the surface on the surface temperature. A strong orientation dependency was shown when state-selected and oriented NO molecules scatter with $\mathrm{Ag}(111)^{32,33}, \mathrm{Pt}(111)^{34}$ and $\mathrm{Ru}-\mathrm{H}^{35}$ surfaces. Steric effects in the scattering of oriented $\mathrm{CH}_{3} \mathrm{~F}$ by graphite were measured ${ }^{36}$, and propensities for inversion symmetry change from antisymmetric to symmetric have been observed for the scattering of hexapole state-selected $\mathrm{ND}_{3}$ molecules with a graphite surface ${ }^{37}$. Wodtke and co-workers combined hexapole focusing with stimulated emission pumping to produce vibrationally highly excited NO radicals, revealing the breakdown of the Born-Oppenheimer approximation in moleculesurface interactions ${ }^{38,39}$.

Hexapolar field geometries have also been used to confine molecules in molecular storage rings or synchrotrons ${ }^{40,41}$. In such a storage ring, the six hexapole rods are bent into a torus of typically half a meter diameter. A packet of ammonia molecules, decelerated to a velocity of about $100 \mathrm{~m} / \mathrm{s}$, can be injected into the ring and stored in near-circular orbits for times up to seconds ${ }^{42}$. Molecular synchrotrons consisting of multiple hexapole segments separated by a small gap have been engineered that allow for a complete confinement of the molecules, both in the longitudinal and transverse directions. Recently, Zieger et al. demonstrated a molecular synchrotron consisting of 40 straight hexapole segments. 
Up to 19 packets of state-selected $\mathrm{ND}_{3}$ molecules were made to propagate through the ring over 1000 times, corresponding to a flight distance for each packet of over a mile ${ }^{43}$.

The ability to efficiently manipulate molecular trajectories inside an electrostatic hexapole depends mainly on the Stark effect and mass of the molecular species of interest and the maximum electric field strength that can be obtained between the hexapole electrodes. The latter is in most hexapole designs limited to about $100 \mathrm{kV} / \mathrm{cm}$. In particular for species with a small effective dipole moment such as nitric oxide (NO), hexapole state selectors of several meters in length are required to focus the beam, limiting the density of the focused beam that can be obtained. Here, we report on a novel mechanical design for an electrostatic hexapole state-selector that allows for a maximum electric field strength of $260 \mathrm{kV} / \mathrm{cm}$, reducing significantly the length of the hexapole that is required to focus the beam. We demonstrate the focusing of a molecular beam of NO radicals $\left(X^{2} \Pi_{1 / 2}, v=0, J=1 / 2, f\right)$ using a hexapole of only $30 \mathrm{~cm}$ length. A beamstop is integrated inside the hexapole, effectively blocking the carrier gas of the molecular beam at minimum loss of NO density. The performance of the hexapole state-selector is investigated by state-selective laser induced fluorescence detection, as well as by two dimensional imaging of the focused packet of NO radicals. Our hexapole design has significant advantages over commonly used designs, in particular in experiments where state-selected molecular beams with optimal density and minimum pollution due to carrier gas atoms are required. These include, for instance, studies of rotational energy transfer between two state-selected molecular beams that have recently become possible using the hexapole reported here ${ }^{44}$.

This paper is organized as follows: in section II we summarize the operation principles of a hexapole state selector, and derive the equations of motion for molecules travelling inside a hexapole. In section III we describe in detail our new hexapole design, focusing on the mechanical details and construction. In sections IV and V we describe experiments on the focusing of NO radicals that were performed to characterize the performance of the state selector.

\section{HEXAPOLE FOCUSING AND STATE SELECTION}

In an inhomogeneous electric field, polar molecules experience a quantum state-specific force due to the Stark effect. Depending on their quantum state, the Stark energy either increases or decreases as the electric field strength increases. The former states are called low-field seeking states, and molecules in these states are driven to regions in space where the electric field strength is minimal. The latter states are called high-field seeking, and molecules in these states experience a force towards regions of maximum electric field.

Focusing of molecules in low and high-field seeking

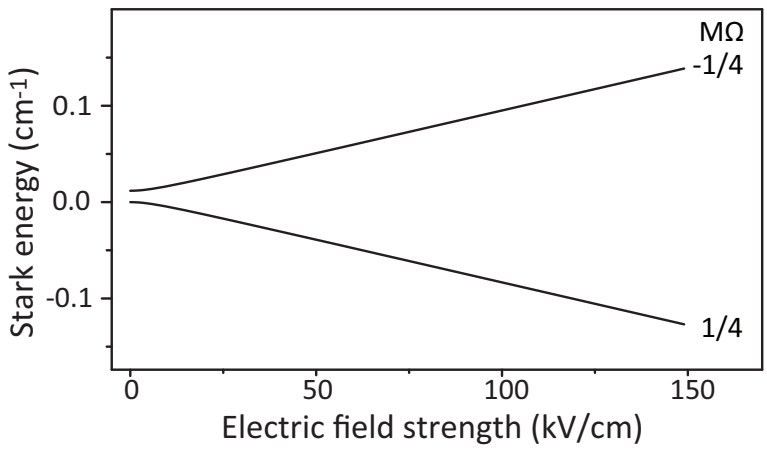

FIG. 1. Stark energy diagram for NO radicals in the $X^{2} \Pi_{1 / 2}, v=0, J=1 / 2$ rotational ground state. Below $15 \mathrm{kV} / \mathrm{cm}$ the Stark shift is quadratic due to the nonzero value for $E_{\Lambda}$ of $318.6 \mathrm{MHz}$. For electric fields above about 15 $\mathrm{kV} / \mathrm{cm}$, however, the Stark shift becomes linear.

states requires an electrode geometry that produces a minimum and maximum of electric field strength at the molecular beam axis, respectively. Whereas focusing of high-field seeking states is only possible using time-varying electric fields (Maxwell's equations forbid a global electric field maximum with static field alone $)^{45-51}$, static electric fields created by straightforward electrode geometries can be used to focus molecules in low-field seeking states.

Most focusing devices for molecules in low-field seeking states consist of four (quadrupole geometry ${ }^{52-54}$ ) or six (hexapole geometry ${ }^{2-4}$ ) electric field electrodes that are placed symmetrically around and parallel to the molecular beam axis. Adjacent electrodes are kept at a voltage difference of several kilovolts, resulting in zero electric field strength at the molecular beam axis, and an increasing electric field strength $E$ as a function of the distance $r$ from the beam axis. For a quadrupole geometry $E$ depends linearly on $r$, whereas for a hexapole geometry $E$ depends quadratically on $r$. The force $\vec{F}$ experienced by the molecules inside the quadrupolar or hexapolar field depends on the gradient of the Stark interaction energy $W_{\text {Stark }}$ :

$$
\vec{F}=-\vec{\nabla} W_{\text {Stark }}
$$

Harmonic restoring forces, resulting in sinusoidal trajectories, are therefore obtained for molecules with a predominantly quadratic and linear Stark shift using the quadrupole and hexapole geometry, respectively.

The Stark shift of molecules like NO in a ${ }^{2} \Pi_{\Omega}$ electronic state is given by

$$
W_{\text {Stark }}=\frac{E_{\Lambda}}{2} \pm \sqrt{\left(\frac{E_{\Lambda}}{2}\right)^{2}+\left(\mu E \frac{M_{J} \Omega}{J(J+1)}\right)^{2}}
$$

where $E_{\Lambda}$ is the zero-field energy splitting between the two $\Lambda$-doublet components of the rotational state $J, \mu$ is the electric dipole moment of the molecule $(\mu=0.16 \mathrm{D}$ for NO), and $\Omega$ and $M_{J}$ are the projections of $J$ onto 

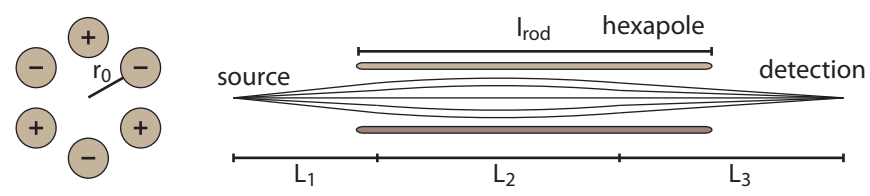

FIG. 2. Left: Schematic representation of the six rods of a hexapole. The voltage $\pm V_{0}$ is applied to the rods in an alternating order. The inner radius $r_{0}$ of the hexapole spans from the center to the surface of the rods. Right: Schematic representation of the focusing curves of a hexapole, from the source to the focus. The first free-flight region, the focusing region and the second free-flight region are indicated by $L_{1}$, $L_{2}$, and $L_{3}$, respectively.

the internuclear and space-fixed axes, respectively. The plus and minus signs in equation 2 apply to the upper and lower $\Lambda$-doublet components, respectively. In Figure 1, the Stark energy diagram for NO radicals in the $X^{2} \Pi_{1 / 2}, v=0, J=1 / 2$ rotational ground state is shown. It is seen that for low electric fields the Stark shift is quadratic due to the nonzero value for $E_{\Lambda}$ of $318.6 \mathrm{MHz}^{55}$. For electric fields above about $15 \mathrm{kV} / \mathrm{cm}$, however, the Stark shift becomes linear. As the majority of the molecules follow trajectories inside the hexapole where the electric field is large, a hexapole geometry is best suited to focus a beam of $\mathrm{NO}$ radicals.

To create the ideal hexapole field, electrodes with hyperbolic shapes should be used ${ }^{56}$. A good approximation to this ideal field can be achieved by using cylindrical electrodes instead. The electrode geometry that is typically used is schematically shown in Figure 2 and consists of six electrodes, hereafter called rods, with a radius $r_{\text {rod }}$ and a length $l_{\text {rod }}{ }^{57}$. The six rods are placed at the outside of a circle with radius $r_{0}$. A voltage difference of $2 V_{0}$ is applied to adjacent rods. The ideal hexapole field is approximated best when $r_{\text {rod }}=0.565 \cdot r_{0}{ }^{58}$.

An ideal hexapole acts as a perfect lens, imaging the molecular packet from the source to the detection region. The focusing properties of the hexapole depend on the electric field strength, and on the time the molecules experience the focusing force. In the hexapole reported here, the voltages are applied to the rods using fast high voltage switches, allowing for a variation of the focusing properties by switching the hexapole field on and off at the appropriate times. Three unique regions are defined along the molecular trajectory, as is shown in Figure 2. In the first region with length $L_{1}$, the molecules progress in free flight from the source to the position where the hexapole voltages are turned on. The second with length $L_{2}$ is defined as the region inside the hexapole for which the molecules experience the focusing force. Finally, the molecules progress in free flight over a distance $L_{3}$ before they reach the detection region. Note that since the voltages on the hexapole are switched, the distances $L_{1}$, $L_{2}$ and $L_{3}$ should not be confused with the physical dimensions of the molecular beamline.

The relation between $L_{1}, L_{2}$, and $L_{3}$ that results in a focus in the detection region can be found in ${ }^{24,59}$. Here, we derive this relation using the so-called matrix method, which is extensively used in accelerator physics ${ }^{60,61}$, and applied to manipulation elements for polar molecules by Crompvoets $^{62}$ and Heiner ${ }^{63}$. In this method, the evolution of the phase-space coordinates of a particle, i.e. its position and velocity, are calculated by transformation matrices. Each region along the trajectory, e.g. a freeflight region or focusing region caused by a hexapole, is represented by a transformation matrix. The full trajectory of the particle is found by multiplying the transformation matrices of each individual region.

We define a coordinate system with its origin located in the molecular beam source. We consider the position $z$ and velocity $v_{z}$ along the beam axis (the longitudinal motion), and use cylindrical coordinates $r$ and $v_{r}$ to denote the phase-space coordinates perpendicular to the beam axis (the transverse motion). For each region, the initial and final phase-space coordinates are represented by the subscripts $i$ and $f$, respectively. Since the hexapole only acts on the radial coordinates, the transformation matrix for the longitudinal motion is given for all three regions by:

$$
\left(\begin{array}{c}
z_{f} \\
v_{z_{f}}
\end{array}\right)=\left(\begin{array}{cc}
1 & t_{k} \\
0 & 1
\end{array}\right) \cdot\left(\begin{array}{c}
z_{i} \\
v_{z_{i}}
\end{array}\right)
$$

where $v_{z_{i}}=v_{z_{f}} \equiv v_{0}$ and $t_{k}$ represents the time the molecule travels in each region, i.e., $t_{k}=L_{k} / v_{0}$ for $k=$ $1,2,3$. For the transverse motion, a similar free-flight transformation matrix applies to regions 1 and 3 .

The transformation matrix $M_{H e x}$ for the transverse motion in region 2 involves the focusing force of the hexapole field. For an ideal hexapole, the electric field strength inside the hexapole is given by

$$
E(r)=3 V_{0} \frac{r^{2}}{r_{0}^{3}}
$$

For molecules with a linear Stark shift that is given by equation 2 (neglecting the $\Lambda$-doublet splitting $E_{\Lambda}$ ), the radial equation of motion follows from equation 1 :

$$
\ddot{r}+\omega^{2} r=0,
$$

with $\omega$ the constant angular frequency

$$
\omega=\sqrt{\frac{\mu}{m} \frac{M_{J} \Omega_{\mathrm{eff}}}{J(J+1)} 6 \frac{V_{0}}{r_{0}^{3}},}
$$

where $m$ is the mass of the focused molecule. The transformation matrix $M_{H e x}$ for the transverse motion in region 2 follows from the motion of molecules in a harmonic potential $^{62}$ :

$$
M_{H e x}=\left(\begin{array}{cc}
\cos \omega t_{2} & \frac{1}{\omega} \sin \omega t_{2} \\
-\omega \sin \omega t_{2} & \cos \omega t_{2}
\end{array}\right) .
$$

The transformation matrix that maps the radial coordinates $r$ and $v$ from the source to the detection region 
is now given by a matrix multiplication of the transformation matrix for each region:

$$
\begin{aligned}
\left(\begin{array}{c}
r_{f} \\
v_{r_{f}}
\end{array}\right)= & \left(\begin{array}{cc}
1 & L_{3} / v_{0} \\
0 & 1
\end{array}\right) \cdot\left(\begin{array}{cc}
\cos \omega L_{2} / v_{0} & \frac{1}{\omega} \sin \omega L_{2} / v_{0} \\
-\omega \sin \omega L_{2} / v_{0} & \cos \omega L_{2} / v_{0}
\end{array}\right) \\
& \cdot\left(\begin{array}{cc}
1 & L_{1} / v_{0} \\
0 & 1
\end{array}\right) \cdot\left(\begin{array}{c}
r_{i} \\
v_{r_{i}}
\end{array}\right) .
\end{aligned}
$$

The relation between $L_{1}, L_{2}$, and $L_{3}$ that results in a focus in the detection region follows from the condition $r_{i}=r_{f}=0$, i.e., assuming a point source for the molecules (see Stolte et $a l^{24}$ ):

$$
L_{3}=\frac{L_{1}+\frac{1}{\kappa} \tan \left(\kappa L_{2}\right)}{L_{1} \kappa \tan \left(\kappa L_{2}\right)-1},
$$

with

$$
\kappa=\frac{\omega}{v_{0}}=\sqrt{\frac{\mu}{m} \frac{M_{J} \Omega_{\mathrm{eff}}}{J(J+1)} 6 \frac{V_{0}}{r_{0}^{3} v_{0}^{2}}} .
$$

In Figure 3 the trajectories are shown that follow from Equation 8 for $\mathrm{NO}$ radicals ${ }^{2} \Pi_{1 / 2}, J=1 / 2, f$ with a velocity of $280 \mathrm{~m} / \mathrm{s}$ that pass through a hexapole. The parameters that are used in this simulation apply to our experimental conditions (vide infra). The molecules originate from a single point with $r_{i}=0$, but ten different radial velocities ranging from $1 \mathrm{~m} / \mathrm{s}$ to $5 \mathrm{~m} / \mathrm{s}$ are simulated. All molecules are seen to focus into a point at a distance of $0.4 \mathrm{~m}$ from the source. It is noted that for $r_{i}>0$ the size of the focus in relation to the size of the source depends on the ratio between $L_{1}$ and $L_{3}$ that is chosen. A 1:1 image is obtained for $L_{1}=L_{3}$.

Equation 9 represents an idealized description of a hexapole. In practice, however, the nozzle is never a point source, and molecules will be produced with a distribution of positions and velocities, blurring the focus. In addition, the $\Lambda$-doublet splitting has been neglected thus far. Close to the beam axis, where the hexapole fields are small, the $\Lambda$-doublet splitting results in a nonlinear Stark effect causing aberrations of the hexapole lens. This effect has been described and analyzed before ${ }^{62,64}$, and results in a reduced transmission of molecules close to the beam axis. In Figure 3 (b) and (c), the influence of the $\Lambda$-doublet splitting and molecular distribution on the focusing properties of the hexapole is shown. The focus is seen to blur when the correct $\Lambda$ doublet splitting and in particular when also a longitudinal velocity spread is assumed.

\section{MECHANICAL IMPLEMENTATION}

The hexapole that is described in this paper is specifically designed to focus a beam of $\mathrm{NO} X^{2} \Pi_{1 / 2}, J=$ $1 / 2, f$ radicals, and has recently been used to inelastically scatter the state-selected packets of NO with packets of Stark-decelerated and state-selected $\mathrm{OH}$ radicals ${ }^{44}$.
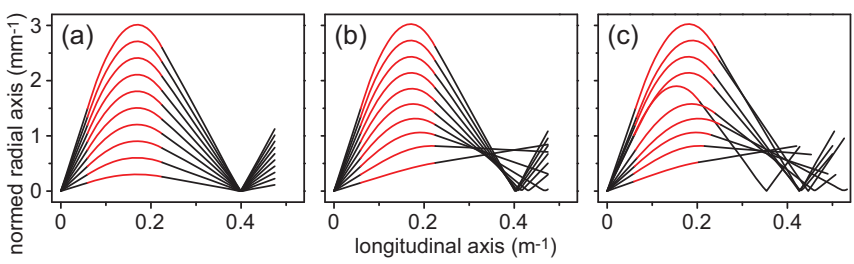

FIG. 3. Calculated focusing curves for NO molecules in the ${ }^{2} \Pi_{1 / 2}, J=1 / 2, f$ state that pass through a hexapole with a velocity of $280 \mathrm{~m} / \mathrm{s}$. The curves are colored black in the first and in the second region of free-flight and red in the focusing region, i.e. the hexapole is turned on/off when the molecules arrive at the position where the curves change color. The simulations are performed assuming (a) zero $\Lambda$-doublet splitting and zero longitudinal velocity spread; (b) zero longitudinal velocity spread but the correct $\Lambda$-doublet splitting for $\mathrm{NO}$ ${ }^{2} \Pi_{1 / 2}, J=1 / 2, f$ of $318.6 \mathrm{MHz}$ is considered; (c) a longitudinal velocity spread of $10 \%$, together with the $\Lambda$-doublet splitting.
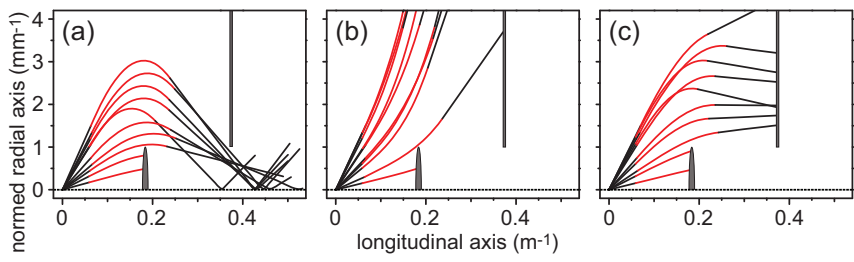

FIG. 4. Calculated focusing curves for NO molecules in (a) the ${ }^{2} \Pi_{1 / 2}, J=1 / 2, f$ state, (b) the ${ }^{2} \Pi_{1 / 2}, J=1 / 2$, e state, and (c) the ${ }^{2} \Pi_{1 / 2}, J=3 / 2, f$ state. In all calculations the molecules have a mean velocity of $280 \mathrm{~m} / \mathrm{s}$, the correct $\Lambda$ doublet splittings are considered and a longitudinal velocity spread of $10 \%$ is assumed. The molecules originate from a single point. Ten different radial velocities from $1 \mathrm{~m} / \mathrm{s}$ to $5 \mathrm{~m} / \mathrm{s}$ are simulated. The curves are colored black in the first and in the second region of free-flight and red in the focusing region, i.e. the hexapole is turned on/off when the molecules arrive at the position where the curves change color. The beamstop and diaphragm are indicated by the shaded structures at the geometric center and downstream from the exit of the hexapole, respectively.

Apart from focusing a beam of NO into the interaction region, additional requirements exist to the hexapole that has been essential to the success of the fore mentioned scattering experiment ${ }^{44}$ :

I. The hexapole needs to be as short as possible to maintain a particle density in the focused packet of NO that is as high as possible;

II. The carrier gas atoms from the beam source should be prevented from entering the interaction region;

III. The state purity of the packet of NO should be as high as possible such that inelastic scattering signals are not overshadowed or contaminated by initial population in levels other than the $X^{2} \Pi_{1 / 2}, J=1 / 2, f$ level.

The most challenging part of these requirements is to design a hexapole with a length that is as short as possible. The low dipole moment of NO of only 0.159 Debye requires high electric fields to focus a packet of $\mathrm{NO}$ 
radicals with a relatively short hexapole. In the most frequently used mechanical design for an electrostatic hexapole, the six rods are suspended by a ceramic disk as illustrated in Figure 5(a). In this Figure, all elements that are placed at high voltage of positive and negative polarity are colored red and cyan, respectively, and all insulating components are coloured orange. Although this design allows for a vacuum gap between adjacent rods, and a relatively large distance for the charge to creep from one rod to the other via the ceramic surface, there is an important shortcoming in this design. As is well known in high voltage engineering, electrical breakdown often initiates at the so-called triple junction, i.e., at the interface between insulator, conductor and vacuum. At these triple junctions, the electric field strength is significantly increased compared to what would be expected from the electrode geometry alone. As neighbouring triple junctions are separated only by the shortest distance between adjacent hexapole rods, electrical breakdown already occurs at relatively low electric field strengths inside the hexapole.

Our hexapole design eliminates this bottle neck by separating the suspensions of adjacent hexapole rods, as illustrated in Figure 5(b) and (c). The set of three rods that share the same voltage polarity is mounted, via adjustable support rods, to a stainless steel disk. This allows for the alignment of the three rods with respect to each other. Both sets are separated from each other by three long Macor insulators. Each insulator is connected to the stainless steel disks in recessed mounting holes with round edges of $6 \mathrm{~mm}$ radius of curvature, effectively shielding the triple junctions. The entire structure is placed in two Macor feet that are mounted on a Macor base plate. All insulators are sufficiently long to prevent electrical discharges over the ceramic surface for voltages below $50 \mathrm{kV}$. With this new hexapole design, electrical breakdown can only occur in the vacuum gaps between the hexapole rods. The maximum electric field strength that can be obtained in the hexapole is limited only by the surface quality of the hexapole rods. To ensure a high surface quality all stainless steel parts are in a first step polished in an industrial surface polishing machine. In a second step these parts are polished by hand with an ultra fine polishing paper.

The hexapole reported here is $300 \mathrm{~mm}$ long and the rods have a radius $r_{\text {rod }}$ of $2 \mathrm{~mm}$. The inner radius of the hexapole is $r_{0}=3.54 \mathrm{~mm}$, following the relation $r_{\text {rod }}=0.565 \cdot r_{0}$ recommended by Anderson ${ }^{58}$. The smallest vacuum distance between adjacent rods is $1.54 \mathrm{~mm}$. A voltage difference of up to $36 \mathrm{kV}$ is applied to adjacent rods by placing the two electrode sets at a potential of $\pm 18 \mathrm{kV}$. A high voltage conditioning procedure is applied to reach these voltages without electrical breakdown. During this procedure, the voltage is applied to the hexapole rods while a possible discharge current is limited by $0.5 \mathrm{G} \Omega$ resistors. The hexapole is only used in the experiments at the final voltage if the current has been less than $10 \mathrm{nA}$ during at least 10 minutes. The
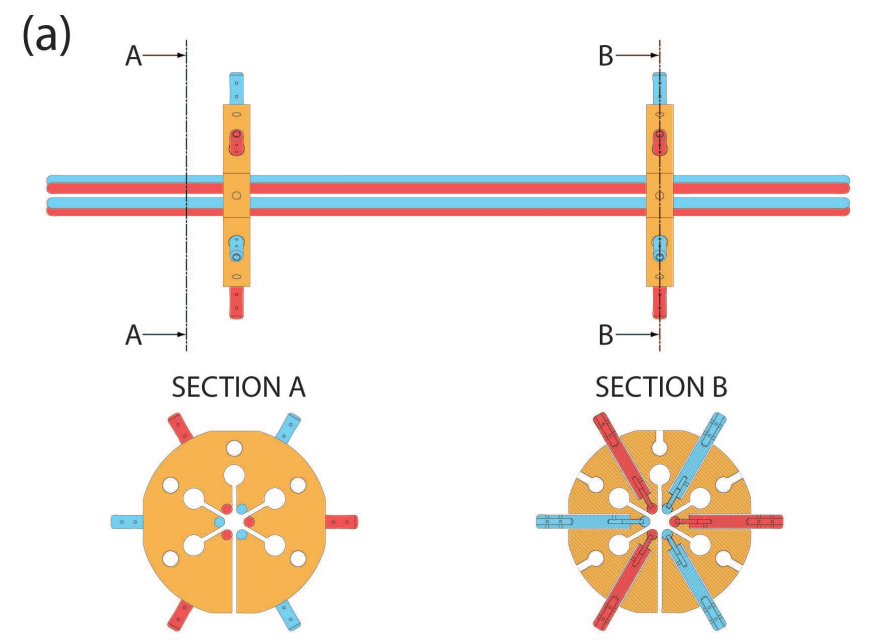

(b)
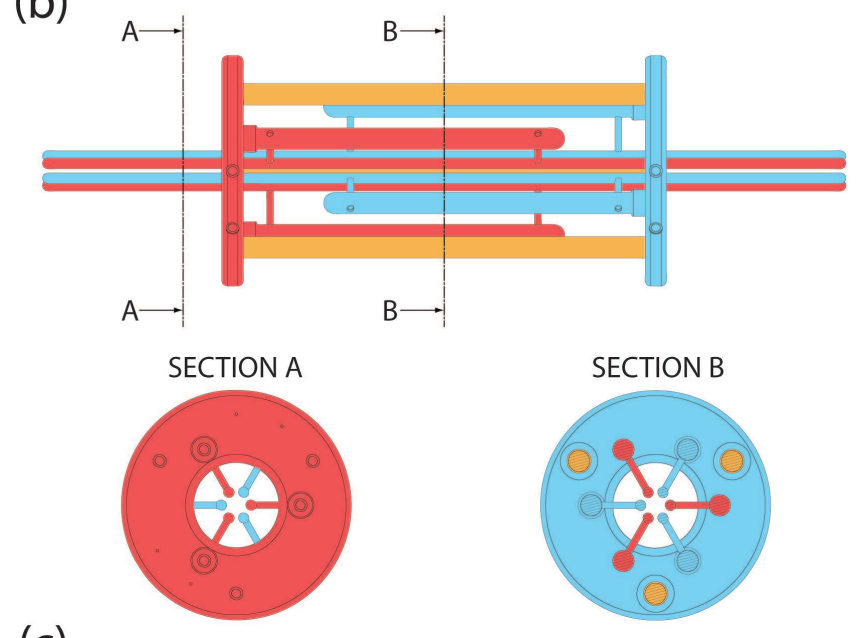

(c)

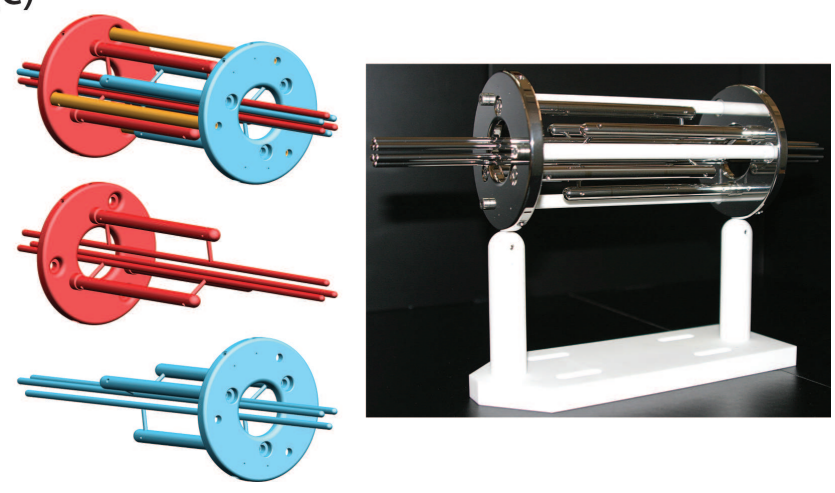

FIG. 5. Technical drawings of hexapole designs. All elements that are placed at high voltage of positive and negative polarity are colored red and cyan, respectively, and all insulating components are coloured orange. (a): Most frequently used mechanical design for an electrostatic hexapole, the six rods are suspended by ceramic disks. (b): Our hexapole design, which separates the suspensions of adjacent hexapole rods. (c): Three dimensional view of the whole hexapole (left,top), and of only three rods and their two suspensions (left,center) and (left,bottom), respectively. The photograph (right) shows the hexapole together with the two Macor feet and the Macor base plate, which are described in the text. 
electric field distribution inside the hexapole is simulated using the COMSOL program ${ }^{65}$, and is shown in Figure 6 (a). At the maximum applied voltage of $\pm 18 \mathrm{kV}$, a maximum electric field strength at the rod's surfaces of $260 \mathrm{kV} / \mathrm{cm}$ is obtained. In most experiments reported here, however, voltages of $\pm 15 \mathrm{kV}$ have been used. These voltages are applied to the hexapole rods using two commercially available transistor based high voltage switches (BEHLKE GmbH).

To filter out the carrier gas atoms (requirement II) and to achieve an almost perfect quantum-state purity of the focused NO packet (requirement III), a $2 \mathrm{~mm}$ diameter beamstop was installed on the molecular beam axis at the geometric center of the hexapole. A $2 \mathrm{~mm}$ diameter diaphragm was positioned between the exit of the hexapole and the interaction region. The molecular beam skimmer, beamstop, and diaphragm combination allows no direct line of sight from the source to the interaction region, effectively hindering the carrier gas atoms from reaching the interaction zone. The beamstopdiaphragm combination offers the additional advantage to greatly improve the state purity of the $\mathrm{NO}$ radical beam. Only NO radicals in the $X^{2} \Pi_{1 / 2}, J=1 / 2, f$ state can effectively pass through the hexapole. This is illustrated in Figure 4 that shows focusing curves for NO molecules in the low-field-seeking $X^{2} \Pi_{1 / 2}, J=1 / 2, f$ rotational ground state (panel (a)), the high-field seeking $X^{2} \Pi_{1 / 2}, J=1 / 2, e$ state (panel (b)), and the low-field seeking $X^{2} \Pi_{3 / 2}, J=3 / 2, f$ rotationally excited state (panel (c)). For the latter simulation, molecules in both the $M \Omega=-3 / 4$ and $M \Omega=-9 / 4$ components are used. It is seen that molecules in quantum states other than the $X^{2} \Pi_{1 / 2}, J=1 / 2, f$ state either hit the beamstop or cannot pass through the diaphragm.

Hexapoles that include beamstops have frequently been employed before ${ }^{23}$. However, most often the beamstop is positioned either in the region between skimmer and entrance of the hexapole, or in the region between the exit of the hexapole and the interaction region. Although mechanically more challenging, the most ideal position of the beamstop for our application is the geometric center of the hexapole. At this position, the molecular trajectories have the largest deviation from the molecular beam axis, resulting in a minimum loss of molecules in the $X^{2} \Pi_{1 / 2}, J=1 / 2, f$ state. In addition, the $\Lambda$ doublet splitting of the $X^{2} \Pi_{1 / 2}, J=1 / 2$ rotational state results in a reduced transmission for molecules travelling close to the molecular beam axis. In our hexapole design, the beamstop is attached to one of the rods, i.e., the beamstop is on high voltage causing locally an asymmetric field distribution. This is illustrated in Figure 6(b) and (c) that shows the simulated field distribution at the longitudinal center of the hexapole, and $5 \mathrm{~mm}$ further downstream, respectively. It is seen that the distortion of the electric field due to the presence of the beamstop is negligible for almost all sections of the hexapole. The focusing properties of the hexapole are therefore expected to be hardly affected by the presence of the beamstop.
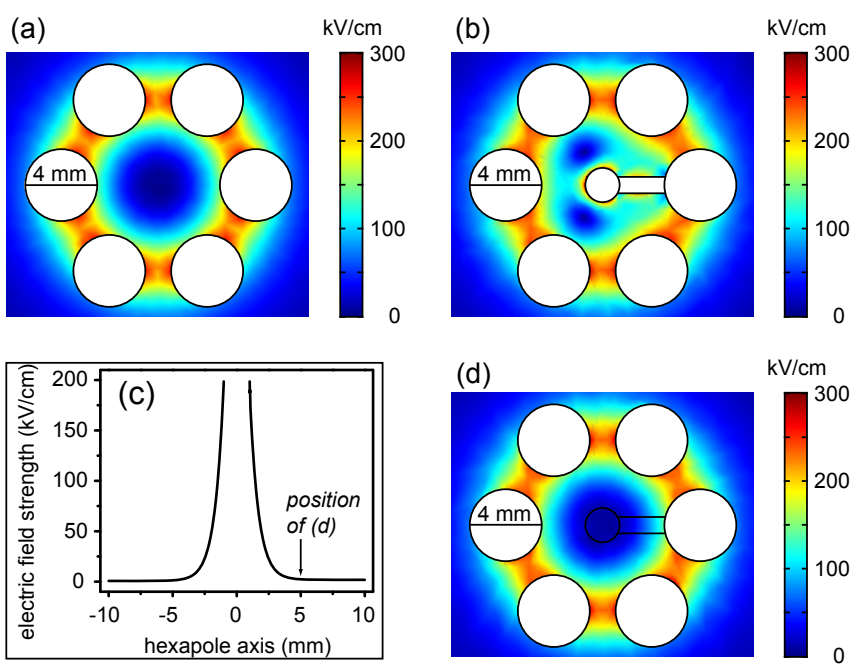

FIG. 6. Two dimensional electric field distribution inside the hexapole at different positions along the molecular beam axis. (a) Field distribution at the center of the hexapole without beamstop; (b) Field distribution at the center of the hexapole with beamstop; (d) Field distribution $5 \mathrm{~mm}$ further downstream from the center of the hexapole; (c) Electric field strength along the molecular beam axis. $\mathrm{x}=0$ is the position of the beamstop. Negative x-values are before the beamstop and positive $\mathrm{x}$-values are further downstream.

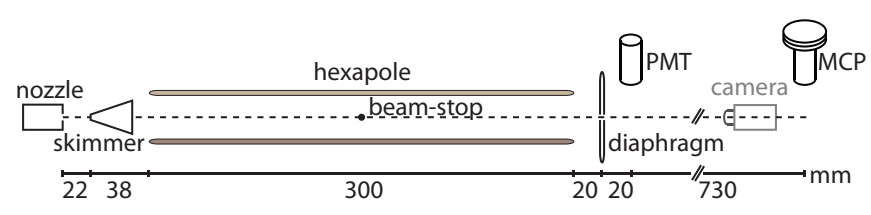

FIG. 7. Schematic representation of the experimental setup. A pulsed beam of NO radicals is passed through a $30 \mathrm{~cm}$ long hexapole. A beamstop is mounted in the geometric center of the hexapole, and a diaphragm is positioned halfway between the exit of the hexapole and the first detection region. The NO radicals are state-selectively detected via Laser Induced Fluorescence in the first detection region $(40 \mathrm{~mm}$ from the exit of the hexapole) and via Resonance Enhanced Multi-Photon Ionization in the second detection region $(730 \mathrm{~mm}$ from the first detection region). The detection laser (not shown) intersects the molecular beam at an angle of 90 degrees. The second detection area can be replaced by a charge-coupled device camera to record the spatial distribution of the NO radicals exiting the hexapole.

Alternatively, the hexapole voltages can be temporarily switched off when the molecular packet is close to the beamstop, effectively producing two successive hexapoles with a grounded beamstop in between.

\section{EXPERIMENT}

The performance of the hexapole was characterized using the experimental setup that is schematically shown in 
Figure 7. A pulsed molecular beam of $\mathrm{NO}$ radicals is produced by expanding a 5\% NO in Xenon (Xe) gas mixture through a $1 \mathrm{~mm}$ diameter commercially available valve (General valves, series 99), using a backing pressure of 2 bar. The nozzle is cooled to $-70^{\circ} \mathrm{C}$, resulting in a mean velocity $v_{0}$ of approximately $300 \mathrm{~m} / \mathrm{s}$. The Gaussian velocity distribution has a width of approximately $15 \%$ (full width at half maximum; FWHM). The NO radicals pass a $2 \mathrm{~mm}$ diameter skimmer that is positioned $22 \mathrm{~mm}$ from the nozzle.

Three different experimental configurations are used to characterize the hexapole. In the first configuration the hexapole is not installed, and the NO molecules are detected after $378 \mathrm{~mm}$ of free flight from the skimmer to the detection region. In the second configuration, the $300 \mathrm{~mm}$ long hexapole is installed $38 \mathrm{~mm}$ downstream from the skimmer, leaving a $40 \mathrm{~mm}$ free flight section from the exit of the hexapole to the detection region. In this configuration, the beamstop and diaphragm are not installed yet. In the third configuration, the $2 \mathrm{~mm}$ diameter beamstop is installed in the center of the hexapole and the $2 \mathrm{~mm}$ diameter diaphragm is installed $20 \mathrm{~mm}$ downstream from the exit of the hexapole. In all experiments a voltage of $\pm 15 \mathrm{kV}$ is applied to the hexapole rods.

The NO radicals are state-selectively detected using saturated laser-induced fluorescence (LIF) using the $0-0$ band of the $A^{2} \Sigma^{+} \leftarrow X^{2} \Pi$ transition around $226 \mathrm{~nm}$ using a pulsed dye laser with a bandwidth of $0.06 \mathrm{~cm}^{-1}$. The fluorescence is either mapped onto a PMT that is located above the detection area, or imaged onto a chargedcoupled device (CCD) camera that is located further downstream from the detection area. The PMT is used to record integral signal intensities, whereas the CCD camera is used to record the spatial distribution of the NO radicals exiting the hexapole. Optionally, the camera can be replaced by a second detection area $730 \mathrm{~mm}$ further downstream from the first detection area (see Figure 7). In this second detection area, the pulsed dye laser is used to detect NO radicals by $(1+1)$ Resonance Enhanced Multi-Photon Ionization (REMPI). Since the distance between the two detection areas is well known, the velocity $v_{0}$ of the molecular packet is determined from time of flight measurements.

\section{RESULTS}

Several experiments were performed to test the performance of the hexapole, using $\mathrm{NO}\left(X^{2} \Pi_{1 / 2}\right)$ radicals as a model system. In particular, experiments were conducted to characterize (i) the focusing behavior of the hexapole, (ii) the state purity of the focused packet of NO radicals, and (iii) the ability to eliminate the carrier gas atoms from the molecular beam.

To characterize the focusing properties of the hexapole, NO radicals in the $X^{2} \Pi_{1 / 2}, J=1 / 2, f$ state are detected in the first and second detection area using LIF

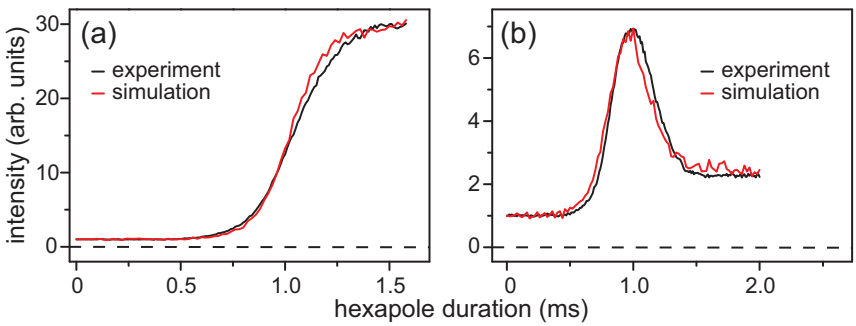

FIG. 8. LIF and REMPI signal intensities of NO $X^{2} \Pi_{1 / 2}, J=$ $1 / 2, f$ radicals recorded in (a) the first and (b) the second detection region, respectively, as a function of the time duration of the high voltage pulse that is applied to the hexapole state selector. The times at which the detection laser is fired are in (a) $1.6 \mathrm{~ms}$ after triggering the molecular beam source (first detection zone) and in (b) $4.0 \mathrm{~ms}$ after triggering the molecular beam source (second detection zone). The measured curves (black) are shown together with the results from three dimensional trajectory simulations (red curves).

and REMPI, respectively. In both detection regions, the time at which the detection laser is fired is chosen to coincide with the peak of the arrival time distribution, i.e., the most intense part of the molecular beam is detected. The hexapole is switched on at the time the molecular beam source is triggered, i.e., the hexapole is already switched to high voltage before the NO radicals arrive at the entrance of the hexapole. The LIF and REMPI signals are recorded as a function of the time duration of the high voltage pulse applied to the hexapole, i.e., the time at which the hexapole is switched off is varied. The beamstop and diaphragm are removed for these measurements in order to observe the inherent focusing properties of the hexapole. The results are shown in Figure 8. In this figure, the times at which the molecules are detected are indicated by vertical arrows.

When the NO radicals are detected in the first detection region, $40 \mathrm{~mm}$ from the exit of the hexapole, an increase in signal is observed as a function of the time duration of the high voltage pulse. The signal reaches a maximum just before the molecules are detected, i.e., the highest density of NO radicals is obtained when the hexapole is switched on during the entire passage of the molecular beam through the hexapole. In this case, the hexapole length and focusing force are just sufficient to focus NO $X^{2} \Pi_{1 / 2}, J=1 / 2, f$ radicals in the first detection region. When the NO radicals are detected in the second detection region, $770 \mathrm{~mm}$ from the exit of the hexapole, an over-focusing effect is observed. The signal intensity reaches a maximum when the hexapole is switched on during approximately $1 \mathrm{~ms}$. When the voltages are applied for longer times, the hexapole focuses too strong, and the NO radicals are focused upstream from the detection region resulting in a decrease in signal intensity.

The results that are obtained from three dimensional numerical trajectory simulations of the experiment are shown in the red curves in Figure 8. In these simu- 
lations, the molecular beam of NO radicals is assumed to have a mean velocity of $v_{0}=300 \mathrm{~m} / \mathrm{s}$, and a longitudinal $\left(\Delta v_{0}\right)$ and transversal $\left(\Delta v_{r}\right)$ Gaussian shaped velocity spread of $15 \%$ and $12 \%$ (FWHM), respectively. The ideal electric field distribution inside the hexapole as given in equation 4 is assumed, and the $\Lambda$-doublet splitting of the $X^{2} \Pi_{1 / 2}, J=1 / 2$ state of $\mathrm{NO}$ is properly taken into account. It is seen that the simulated curves overlap well with the experimentally obtained curves for both experimental configurations, indicating that the focusing properties of the hexapole are well understood. Similar focusing curves were measured and simulated, using exclusively the first detection region, after the beamstop and diaphragm were installed (data not shown). These curves qualitatively resemble the curves shown in Figure 8(a), and show again good overlap with each other. The installation of beamstop and diaphragm resulted in a reduction in maximum signal intensity of only about 10 $20 \%$.

The quantum state purity of the focused packet of NO radicals is investigated spectroscopically. For this, the beamstop and diaphragm are installed, and the hexapole is operated to focus the NO radicals optimally into the first detection region. In Figure 9 two spectra of the $A^{2} \Sigma^{+} \leftarrow X^{2} \Pi$ transition of NO are shown. The black spectrum is taken before the hexapole was installed in the chamber, revealing the original rotational state distribution of the molecular beam, while the red spectrum is recorded with the hexapole, beamstop and diaphragm in place. The rotational lines are labeled using standard spectroscopic nomenclature. It is seen that the majority of the NO radicals in the original molecular beam reside in the $J=1 / 2, f$ and $J=1 / 2$, e levels, although a significant fraction of the NO radicals are found in the $J=3 / 2, f$ and $J=3 / 2$, e rotationally excited levels. When the hexapole, beamstop, and diaphragm assembly is put into use, only NO radicals that reside in the $X^{2} \Pi_{1 / 2}, J=1 / 2, f$ state arrive in the detection region. The spectrum consists of only two lines that both probe the population in the $J=1 / 2, f$ state. It is noted that not only the population in the high-field seeking states of $e$ parity is depleted; also molecules in the rotationally excited states of $f$ parity are effectively blocked by the beamstop-diaphragm combination.

The rotational population distribution is calculated from the measured spectra using the LIFBASE software package ${ }^{66}$. The results are shown in Table I for both spectra. It is seen that the hexapole-beamstopdiaphragm combination greatly reduces the initial population in states other than the $J=1 / 2, f$ state, resulting in a packet of $\mathrm{NO} X^{2} \Pi_{1 / 2}, J=1 / 2, f$ radicals with a state purity approaching $99 \%$. From calibrated LIF measurements, the peak density of the focused packet of NO radicals was determined ${ }^{44}$ to be $9 \pm 3 \times 10^{10} \mathrm{~cm}^{-3}$.

The transmission of NO radicals through the hexapole is investigated further by recording two dimensional fluorescence images of the NO radicals exiting the hexapole. For this, a UV sensitive CCD camera is installed such

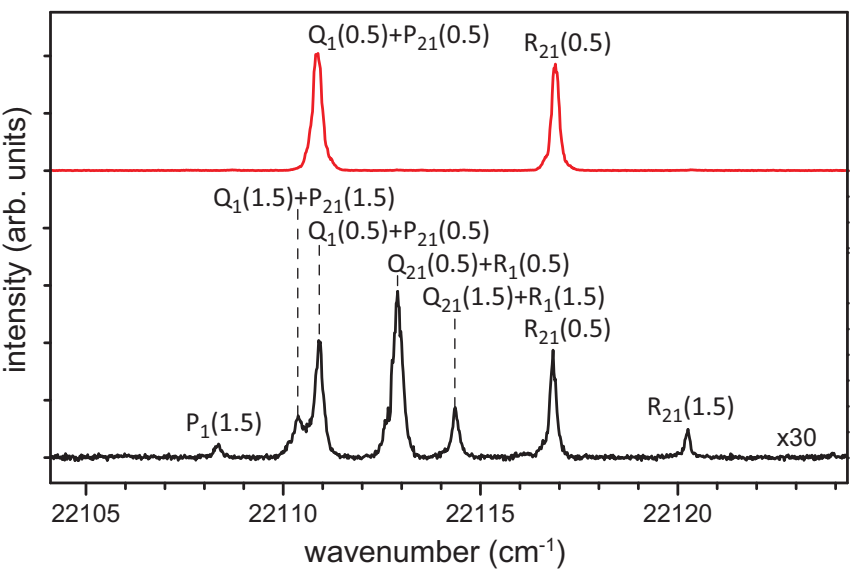

FIG. 9. Spectra of the $A^{2} \Sigma^{+} \leftarrow X^{2} \Pi$ transition of NO around $226 \mathrm{~nm}$. The black spectrum is taken before the hexapole is installed in the chamber; the red spectrum is obtained with the hexapole, beamstop and diaphragm in use. The different rotational transitions are labeled using standard spectroscopic nomenclature.

\begin{tabular}{|c|c|c|}
\hline state & without hexapole (\%) & with hexapole (\%) \\
\hline$J=1 / 2, f$ & $(36.2 \pm 0.5)$ & $(98.8 \pm 0.2)$ \\
$J=1 / 2, e$ & $(36.2 \pm 0.5)$ & $(0.3 \pm 0.2)$ \\
$J=3 / 2, f$ & $(12.4 \pm 0.5)$ & $(0.8 \pm 0.2)$ \\
$J=3 / 2, e$ & $(12.4 \pm 0.5)$ & $(0.1 \pm 0.2)$ \\
$J=5 / 2, f$ & $(1.4 \pm 0.5)$ & $(0.0 \pm 0.2)$ \\
$J=5 / 2, e$ & $(1.4 \pm 0.5)$ & $(0.0 \pm 0.2)$ \\
\hline
\end{tabular}

TABLE I. Rotational population distribution corresponding to the spectra shown in Figure 9. The populations are calculated from the measured spectra using the LIFBASE $\operatorname{program}^{66}$.

that the transverse distribution of the $\mathrm{NO}$ radicals in the first detection zone is imaged. In Figure 10 several images are shown that are taken for NO radicals in different quantum states. In the images (a) to (d) the hexapolebeamstop-diaphragm combination is in use, whereas for the images (e) to $(\mathrm{h})$ the diaphragm was removed while the beamstop was kept in place.

Images (a) and (e) show the transverse distribution for NO radicals in the low-field seeking $J=1 / 2, f$ rotational ground state that is measured with and without the diaphragm, respectively. It is seen that the packet of NO is focused through the diaphragm resulting in a spherically symmetric molecular distribution. The size of the distribution is slightly larger when the diaphragm is removed, indicating that the wings of the NO packet are clipped by the diaphragm. In the images (b) and (f) the distributions are shown that are recorded for NO radicals in the high-field seeking $J=1 / 2$, e state. Molecules in this state are deflected from the molecular beam axis, as can clearly been seen in image (f) where the fluorescence appears only on the side of the image. The diaphragm prevents these molecules from arriving in the detection region, leading to zero signal in image (b). In the images (c) 

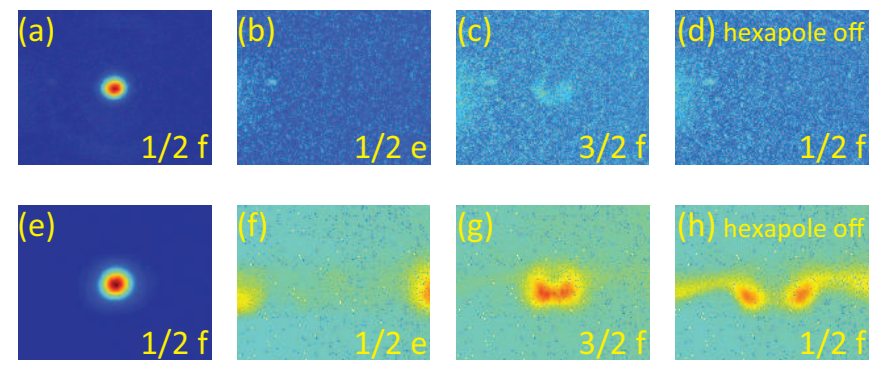

FIG. 10. Fluorescence images of NO radicals in various quantum states that are recorded with the charge-coupled device camera. In the images (a) to (d) the hexapole, beamstop and diaphragm are in use. In the images (e) to (h) the beamstop is in place but the diaphragm is removed. The molecular state that is used is indicated in each image. In the images (d) and (h) the hexapole voltages are turned off.

and $(\mathrm{g})$ the distributions are shown for NO radicals in the low-field seeking $J=3 / 2, f$ state. These molecules are focused towards the molecular beam axis, but the force acting on the molecules is too weak to focus the molecules around the beamstop and through the diaphragm. This is clearly visible in image $(\mathrm{g})$ where a two-component distribution is shown in which the shadow of the beamstop is clearly recognized. Again, a properly positioned diaphragm will eliminate most of these molecules from the beam, greatly improving the quantum state purity of the transmitted packet of molecules.

Finally, we investigate the ability of the hexapolebeamstop-diaphragm combination to eliminate the carrier gas atoms from the beam. Unfortunately, the carrier gas atoms cannot be detected and imaged directly, but we can use NO $J=1 / 2, f$ radicals as a proxy for the carrier gas atoms if we apply no voltages to the hexapole. The NO radicals then progress in a straight path from the nozzle to the detection, i.e. they behave just like carrier gas atoms that experience no forces in the hexapole. Images (d) and (h) in Figure 10 show the distributions that are recorded for NO radicals in the $J=1 / 2, f$ state when the diaphragm is installed and removed, respectively. Image (h) illustrates the blocking of the carrier gas atoms by the beamstop. No fluorescence signal is recorded at the center of the image. The streak of molecules that is detected on both sides of the beamstop's shadow originates from molecules that pass between neighboring hexapole rods. When the diaphragm is in place (image (d)), no molecules are detected, illustrating that the beamstopdiaphragm combination effectively blocks all carrier gas atoms.

\section{CONCLUSION}

We have presented a novel design for an electrostatic hexapole state selector to focus polar molecules in a molecular beam. The design allows for electric fields up to $260 \mathrm{kV} / \mathrm{cm}$, reducing the length of the hexapole that is needed to focus the beam. A beamstop is integrated inside the hexapole, effectively filtering out the carrier gas atoms from the beam at minimum loss of beam density. The focusing of a molecular beam of NO $X^{2} \Pi_{1 / 2}, J=1 / 2, f$ radicals is demonstrated using a hexapole of only $30 \mathrm{~cm}$ length. The focused packet of NO is characterized by state-selective laser induced fluorescence detection, as well as by two dimensional imaging using a UV sensitive CCD camera. The resulting packet of NO radicals has a state purity approaching $99 \%$ and a density of $9 \pm 3 \cdot 10^{10} \mathrm{~cm}^{-3}$.

Our hexapole design has significant advantages over commonly used designs in experiments where molecular beams with optimal density and quantum state purity are required, in particular for molecular species with a small to moderate Stark effect. The reduction of carrier gas atom density to insignificant values is essential in scattering experiments where the carrier gas atoms may pollute the target or induce unwanted scattering signals. Our hexapole is easy to build and to implement, and is well suited for a variety of molecular beam experiments ranging from crossed-beam scattering studies to surface scattering experiments.

${ }^{1}$ G. Scoles, ed., Atomic and molecular beam methods, Vol. 1 \& 2 (Oxford University Press, New York, NY, USA, 1988 \& 1992).

${ }^{2}$ K. H. Kramer and R. B. Bernstein, J. Chem. Phys. 42, 767 (1965).

${ }^{3}$ P. R. Brooks and E. M. Jones, J. Chem. Phys. 45, 3449 (1966).

${ }^{4}$ R. J. Beuhler, R. B. Bernstein, and K. H. Kramer, J. Am. Chem. Soc. 88, 5331 (1966).

${ }^{5}$ P. R. Brooks, Science 193, 11 (1976).

${ }^{6}$ M. Kurahashi and Y. Yamauchi, Phys. Rev. A 78, 022708 (2008).

${ }^{7}$ M. Kurahashi and Y. Yamauchi, Rev. Sci. Instrum. 80, 083103 (2009).

${ }^{8}$ S. Stolte, Ber. Bunsenges. Phys. Chem. 86, 413 (1982).

${ }^{9}$ D. H. Parker and R. B. Bernstein, Annu. Rev. Phys. Chem. 40, 561 (1989).

${ }^{10}$ H. Jalink, D. H. Parker, and S. Stolte, J. Chem. Phys. 85, 5372 (1986).

${ }^{11}$ M. A. Weibel, T. D. Hain, and T. J. Curtiss, J. Chem. Phys. 108, 3134 (1998).

${ }^{12}$ T. D. Hain, R. M. Moision, and T. J. Curtiss, J. Chem. Phys. 111, 6797 (1999).

${ }^{13}$ A. Schiffman and D. W. Chandler, Int. Rev. Phys. Chem. 14, 371 (1995).

${ }^{14}$ D. Watanabe, H. Ohoyama, T. Matsumura, and T. Kasai, Phys. Rev. Lett. 99, 043201 (2007).

${ }^{15}$ D. H. Parker, H. Jalink, and S. Stolte, J. Phys. Chem. 91, 5427 (1987).

${ }^{16}$ C. J. Eyles, M. Brouard, C. H. Yang, J. Kłos, F. J. Aoiz, A. Gijsbertsen, A. E. Wiskerke, and S. Stolte, Nature Chem. 3, 597 (2010).

${ }^{17}$ J. J. van Leuken, F. H. W. Van Amerom, J. Bulthuis, J. G. Snijders, and S. Stolte, J. Phys. Chem. 99, 15573 (1995).

${ }^{18}$ A. Gijsbertsen, H. Linnartz, G. Rus, A. E. Wiskerke, S. Stolte, D. W. Chandler, and J. Kłos, J. Chem. Phys. 123, 224305 (2005).

${ }^{19}$ A. Gijsbertsen, H. Linnartz, and S. Stolte, J. Chem. Phys. 125, 133112 (2006).

${ }^{20}$ K. Schreel, J. Schleipen, A. Eppink, and J. J. ter Meulen, J. Chem. Phys. 99, 8713 (1993).

${ }^{21}$ K. Schreel and J. J. ter Meulen, J. Chem. Phys. 105, 4552 (1996).

${ }^{22}$ M. C. van Beek, G. Berden, H. L. Bethlem, and J. J. ter Meulen, Phys. Rev. Lett. 86, 4001 (2001). 
${ }^{23}$ M. C. van Beek, J. J. ter Meulen, and M. H. Alexander, J. Chem. Phys. 113, 628 (2000).

${ }^{24}$ S. Stolte, J. Reuss, and H. Schwartz, Physica 57, 254 (1972).

${ }^{25}$ S. Stolte, J. Reuss, and H. Schwartz, Physica 66, 211 (1973).

${ }^{26}$ H. Thuis, S. Stolte, and J. Reuss, Chem. Phys. 43, 351 (1979).

${ }^{27}$ H. Kohguchi and T. Suzuki, Annu. Rep. Prog. Chem. 98, 421 (2002).

${ }^{28} \mathrm{M}$. Brouard and C. Vallance, eds., Tutorials in molecular reaction dynamics (Royal Society of Chemistry, 2010).

${ }^{29}$ T. D. Hain, M. A. Weibel, K. M. Backstrand, and T. J. Curtiss, J. Phys. Chem. A 101, 7674 (1997).

${ }^{30}$ B. J. Bichsel, M. A. Morrison, N. Shafer-Ray, and E. R. I. Abraham, Phys. Rev. A 75, 023410 (2007).

${ }^{31}$ L. V. Novakoski and G. M. McClelland, Phys. Rev. Lett. 59, 1259 (1987).

${ }^{32}$ E. W. Kuipers, M. G. Tenner, A. W. Kleyn, and S. Stolte, Nature 334, 420 (1988).

${ }^{33}$ M. G. Tenner, E. W. Kuipers, A. W. Kleyn, and S. Stolte, J. Chem. Phys. 89, 6552 (1988).

${ }^{34}$ E. W. Kuipers, M. G. Tenner, A. W. Kleyn, and S. Stolte, Phys. Rev. Lett. 62, 2152 (1989).

${ }^{35}$ B. Berenbak, B. Riedmüller, S. Stolte, and A. W. Kleyn, Chem. Phys. 301, 309 (2004).

${ }^{36}$ T. J. Curtiss and R. B. Bernstein, J. Chem. Phys. 161, 212 (1989).

37 T. J. Curtiss, R. S. Mackay, and R. B. Bernstein, J. Chem. Phys. 93, 7387 (1990).

${ }^{38}$ D. Matsiev, J. Chen, M. Murphy, and A. M. Wodtke, J. Chem. Phys. 118, 9477 (2003).

${ }^{39}$ J. Chen, D. Matsiev, J. D. White, M. Murphy, and A. M. Wodtke, Chem. Phys. 301, 161 (2004).

${ }^{40}$ F. M. H. Crompvoets, H. L. Bethlem, R. T. Jongma, and G. Meijer, Nature 411, 174 (2001).

${ }^{41}$ F. M. H. Crompvoets, H. L. Bethlem, J. Küpper, A. J. A. van Roij, and G. Meijer, Phys. Rev. A 69, 063406 (2004).

${ }^{42}$ C. E. Heiner, D. Carty, G. Meijer, and H. L. Bethlem, Nature Phys. 3, 115 (2007).

${ }^{43}$ P. C. Zieger, S. Y. T. van de Meerakker, C. E. Heiner, H. L. Bethlem, A. J. A. van Roij, and G. Meijer, Phys. Rev. Lett. 105, 173001 (2010).

${ }^{44}$ M. Kirste, X. Wang, H. C. Schewe, G. Meijer, K. Liu, A. van der Avoird, L. M. C. Janssen, K. B. Gubbels, G. C. Groenenboom, and S. Y. T. van de Meerakker, Science 338, 1060 (2012).
${ }^{45}$ E. D. Courant and H. S. Snyder, Ann. Phys. 3, 1 (1958).

${ }^{46}$ D. Auerbach, E. E. A. Bromberg, and L. Wharton, J. Chem. Phys. 45, 2160 (1966)

${ }^{47}$ D. Kakati and D. C. Lainé, Phys. Lett. A 24, 676 (1967).

${ }^{48}$ H. L. Bethlem, M. R. Tarbutt, J. Küpper, D. Carty, K. Wohlfart, E. A. Hinds, and G. Meijer, J. Phys. B 39, R263 (2006).

${ }^{49}$ T. Junglen, T. Rieger, S. A. Rangwala, P. W. H. Pinkse, and G. Rempe, Phys. Rev. Lett. 92, 223001 (2004).

${ }^{50}$ F. Filsinger, U. Erlekam, G. von Helden, J. Küpper, and G. Meijer, Phys. Rev. Lett. 100, 133003 (2008).

${ }^{51}$ S. Putzke, F. Filsinger, H. Haak, J. Küpper, and G. Meijer, Phys. Chem. Chem. Phys. 13, 18962 (2011).

52 J. P. Gordon, H. J. Zeiger, and C. H. Townes, Phys. Rev. 95, $282(1954)$.

${ }^{53}$ J. P. Gordon, H. J. Zeiger, and C. H. Townes, Phys. Rev. 99, 1264 (1955).

${ }^{54}$ H. G. Bennewitz, W. Paul, and C. Schlier, Z. Phys. 141, 6 (1955).

${ }^{55}$ W. L. Meerts and A. Dymanus, J. Mol. Spectr. 44, 320 (1972).

${ }^{56}$ F. M. H. Crompvoets, H. L. Bethlem, and G. Meijer, Adv. At., Mol., Opt. Phys. 52, 209 (2006).

${ }^{57} \mathrm{~J}$. Reuss, in Atomic and molecular beam methods, Vol. 1, edited by G. Scoles (Oxford University Press, New York, NY, USA, 1988) Chap. 11, pp. 276-292.

${ }^{58} \mathrm{R}$. W. Anderson, J. Phys. Chem. A 101, 7664 (1997).

${ }^{59} \mathrm{~A}$. Gijsbertsen, Towards a better understanding of inelastic atom-molecule collisions, $\mathrm{Ph}$. D. thesis, Vrije Universiteit, Amsterdam, The Netherlands (2006).

${ }^{60}$ S. Y. Lee, Accelerator physics, 2nd ed. (World Scientific, Singapore, 2004).

${ }^{61}$ A. P. Banford, The transport of charged particle beams (London: E. \& F. N. Spon Limited, 1966).

${ }^{62}$ F. M. H. Crompvoets, A storage ring for neutral molecules, $\mathrm{Ph}$. D. thesis, Radboud Universiteit, Nijmegen, The Netherlands (2005).

${ }^{63}$ C. E. Heiner, A molecular synchrotron, Ph. D. thesis, Radboud Universiteit, Nijmegen, The Netherlands (2009).

${ }^{64}$ R. T. Jongma, T. Rasing, and G. Meijer, J. Chem. Phys. 102, 1925 (1995).

${ }^{65}$ COMSOL, "COMSOL Multiphysics software (Version 3.2)", COMSOL, Inc. US, http://www. comsol.com (2006).

${ }^{66}$ J. Luque and D. R. Crosley, "LIFBASE: Database and spectral simulation program (Version 1.5)", SRI International Report MP 99-009 (1999). 